\title{
Small-x effects in forward-jet production at HERA
}

\author{
Cyrille Marquet \\ Service de physique théorique, CEA/Saclay, 91191 Gif-sur-Yvette cedex, France \\ URA 2306, unité de recherche associée au CNRS
}

\begin{abstract}
We investigate small- $x$ effects in forward-jet production at HERA in the two-hard-scale region $k_{T} \sim Q \gg \Lambda_{Q C D}$. We show that, despite describing different energy regimes, both a BFKL parametrization and saturation parametrizations describe well the H1 and ZEUS data for $d \sigma / d x$ published a few years ago. This is confirmed when comparing the predictions to the latest data.
\end{abstract}

\section{INTRODUCTION}

Forward-jet production is a process in which a virtual photon strongly interacts with a proton and a jet is detected in the forward direction of the proton. The virtuality of the photon $Q^{2}$ and the squared transverse momentum of the jet $k_{T}^{2}$ are hard scales of about the same magnitude. In the Regge limit of perturbative QCD, i.e. when the centre-of-mass energy in a collision is much bigger than the fixed hard scales of the problem, the scattering amplitudes grow with increasing energy as described by the BFKL equation [1]. The forward-jet measurement was originally proposed [2] to test the BFKL equation because, if the energy in the photon-proton collision $W$ is large enough, it lies in the kinematic regime corresponding to the Regge limit $\left(W^{2} \gg Q^{2}\right)$.

The question is whether the BFKL equation is relevant at the present energies, or if usual perturbative QCD in the Bjorken limit is still sufficient to describe the data. We adress that problem by computing the forward-jet cross-section in the high-energy regime and by comparing the BFKL predictions with the available data. We also adress the problem of saturation [3]: it is well-known that the BFKL growth is damped by saturation effects when energies become too high and the scattering amplitudes approach the unitatity limit. We implement saturation effects in a very simple way, inspired by the Golec-Biernat and Wüsthoff approach [4] and check the consistency with the data.

\section{FORMULATION}

The QCD cross-section for forward-jet production in a lepton-proton collision reads

$$
\frac{d^{(4)} \sigma}{d x d Q^{2} d x_{J} d k_{T}^{2}}=\frac{\alpha_{e m}}{\pi x Q^{2}}\left\{\left(\frac{d \sigma_{T}^{\gamma * p \rightarrow J X}}{d x_{J} d k_{T}^{2}}+\frac{d \sigma_{L}^{\gamma * p \rightarrow J X}}{d x_{J} d k_{T}^{2}}\right)(1-y)+\frac{d \sigma_{T}^{\gamma * p \rightarrow J X}}{d x_{J} d k_{T}^{2}} \frac{y^{2}}{2}\right\},
$$

where $x$ and $y$ are the usual kinematic variables of deep inelastic scattering and $Q^{2}$ is the virtuality of the intermediate photon that undergoes the hadronic interaction. 
$d \sigma_{T, L}^{\gamma * p \rightarrow J X} / d x_{J} d k_{T}^{2}$ is the cross-section for forward-jet production in the collision of this transversally $(\mathrm{T})$ or longitudinally $(\mathrm{L})$ polarized virtual photon with the target proton. $k_{T}$ is the jet transverse momentum and $x_{J}$ its longitudinal momentum fraction with respect to the proton.

Let us now consider the high-energy regime: $x=\log \left(Q^{2} /\left(Q^{2}+W^{2}\right)\right) \ll 1$. In an appropriate frame called the dipole frame, the virtual photon undergoes the hadronic interaction via a fluctuation into a colorless $q \bar{q}$ pair, a dipole. The squared wavefunctions $\phi_{T}^{\gamma}$ and $\phi_{L}^{\gamma}$ describing the splitting of the virtual photon onto a dipole are well-known. The dipole then interacts with the target proton and one has the following factorization

$$
\frac{d \sigma_{T, L}^{\gamma * p \rightarrow J X}}{d x_{J} d k_{T}^{2}}=\int_{0}^{\infty} 2 \pi r d r \phi_{T, L}^{\gamma}(r, Q) \frac{d \sigma_{q \bar{q}}}{d x_{J} d k_{T}^{2}}(r)
$$

$d \sigma_{q \bar{q}}(r) / d x_{J} d k_{T}^{2}$ is the cross-section for forward-jet production in the dipole-proton collision. The integration variable $r$ represents the size of the intermediate dipole.

It was shown in [5] that the emission of the forward jet can be described through the interaction of an effective gluonic (gg) dipole:

$$
\frac{d \sigma_{q \bar{q}}}{d x_{J} d k_{T}^{2}}(r)=\frac{\pi N_{c}}{16 k_{T}^{2}} f_{e f f}\left(x_{J}, k_{T}^{2}\right) \int_{0}^{\infty} d \bar{r} J_{0}\left(k_{T} \bar{r}\right) \frac{\partial}{\partial \bar{r}}\left(\bar{r} \frac{\partial}{\partial \bar{r}} \sigma_{(q \bar{q})(g g)}(r, \bar{r}, Y)\right)
$$

with $Y=\log \left(x_{J} / x\right)$ the rapidity assumed very large. $\sigma_{(q \bar{q})(g g)}(r, \bar{r}, Y)$ is the $q \bar{q}$ dipole (size $r$ )-gg dipole ( size $\bar{r}$ ) total cross-section with rapidity Y. As usual, the dipoles emerge as the effective degrees of freedom at high energies: $\sigma_{(q \bar{q})(g g)}$ contains any number of gluon exchanges and therefore this formulation goes beyond $k_{T}$-factorization which assumes only a two-gluon exchange. The effective parton distribution function $f_{\text {eff }}$ is given by: $f_{\text {eff }}\left(x_{J}, k_{T}^{2}\right)=g\left(x_{J}, k_{T}^{2}\right)+C_{F}\left(q\left(x_{J}, k_{T}^{2}\right)+\bar{q}\left(x_{J}, k_{T}^{2}\right)\right) / N_{c}$ where $g$ (resp. $\left.q, \bar{q}\right)$ is the gluon (resp. quark, antiquark) distribution function in the incident proton.

\section{BFKL parametrization}

The BFKL $q \bar{q}$-dipole $g g$-dipole cross-section reads

$$
\sigma_{(q \bar{q})(g g)}^{B F K L}(r, \bar{r}, Y)=2 \pi \alpha_{s}^{2} r^{2} \int \frac{d \gamma}{2 i \pi}\left(\frac{\bar{r}}{r}\right)^{2 \gamma} \frac{\exp \left(\frac{\alpha_{s} N_{c}}{\pi} \chi(\gamma) Y\right)}{\gamma^{2}(1-\gamma)^{2}}
$$

with the complex integral running along the imaginary axis from $1 / 2-i \infty$ to $1 / 2+i \infty$ and with the BFKL kernel given by $\chi(\gamma)=2 \psi(1)-\psi(1-\gamma)-\psi(\gamma)$ where $\psi(\gamma)$ is the logarithmic derivative of the Gamma function. It comes about when the interaction between the $q \bar{q}$-dipole and the $g g$-dipole is restricted to a two-gluon exchange. One can easily show, putting (4) in (2) and (3), that this formulation is equivalent to using $k_{T}$-factorization. We are going to perform a fit of the parametrization (4) to the data. The parameters are $\lambda=4 \alpha_{s} N_{c} \log (2) / \pi$ and a normalization. 

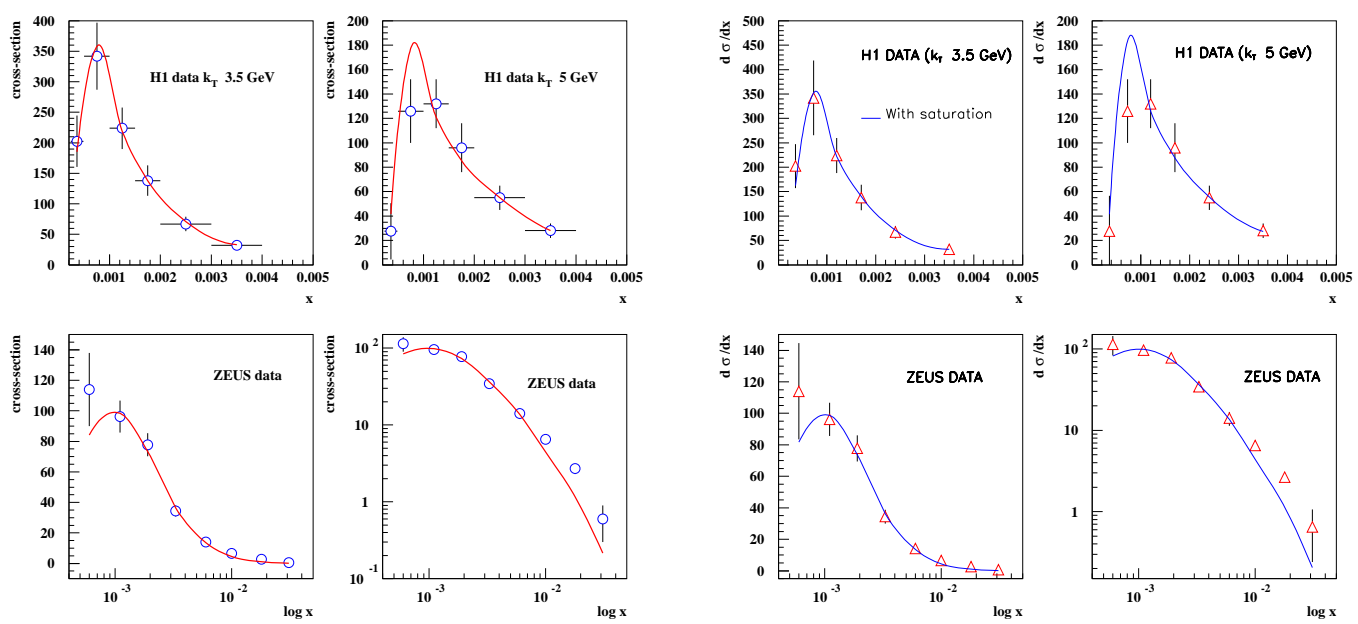

FIGURE 1. Fits to the H1 and ZEUS forward-jet old data for $d \sigma / d x$. The left plot shows the BFKL fit and the right plot shows one of the saturation fits (called sat. in the text).

\section{Saturation parametrization}

To take into account saturation effects, let us consider the following parametrization:

$$
\sigma_{(q \bar{q})(g g)}^{s a t}(r, \bar{r}, Y)=4 \pi \alpha_{s}^{2} \sigma_{0}\left(1-\exp \left(-\frac{r_{\mathrm{eff}}^{2}(r, \bar{r})}{4 R_{0}^{2}(Y)}\right)\right) .
$$

The dipole-dipole effective radius $r_{\mathrm{eff}}^{2}(r, \bar{r})$ is defined through the two-gluon exchange:

$$
4 \pi \alpha_{s}^{2} r_{\mathrm{eff}}^{2}(r, \bar{r}) \equiv \sigma_{(q \bar{q})(g g)}^{B F K L}(r, \bar{r}, 0)=4 \pi \alpha_{s}^{2} \min \left(r^{2}, \bar{r}^{2}\right)\left\{1+\log \frac{\max (r, \bar{r})}{\min (r, \bar{r})}\right\}
$$

while the saturation radius is parametrized by $R_{0}(Y)=e^{-\frac{\lambda}{2}\left(Y-Y_{0}\right)} / Q_{0}$ with $Q_{0} \equiv 1 G e V$. The parameters for the fit are $\lambda, Y_{0}$ and the normalization $\sigma_{0}$.

\section{PHENOMENOLOGY}

To compare the cross-section (1) with the data for $d \sigma / d x$, the three remaining integration are carried out taking into account the different sets of cuts provided by the different experiments. Fits have been performed to the old sets of data [6, 7] for the BFKL parametrization [8] and the saturation parametrization [9]. With all $\chi^{2}$ values of about 1 , the BFKL fit gives $\lambda=0.430$ and the saturation fit shows two $\chi^{2}$ minima for $\left(\lambda=0.402, Y_{0}=-0.82\right)$ (sat.) and $\left(\lambda=0.370, Y_{0}=8.23\right)$ (weak sat.). The plots are shown on Fig1. Despite describing different energy regimes, both a BFKL parametrization and saturation parametrizations describe well the data. The first saturation minima corresponds to a strong saturation effect as, for typical values of $Y$, the saturation scale $1 / R_{0}$ is $5 \mathrm{Gev}$ which is the value of a typical $k_{T}$. The second saturation minima corresponds to small saturation effets and rather describes BFKL physics. 

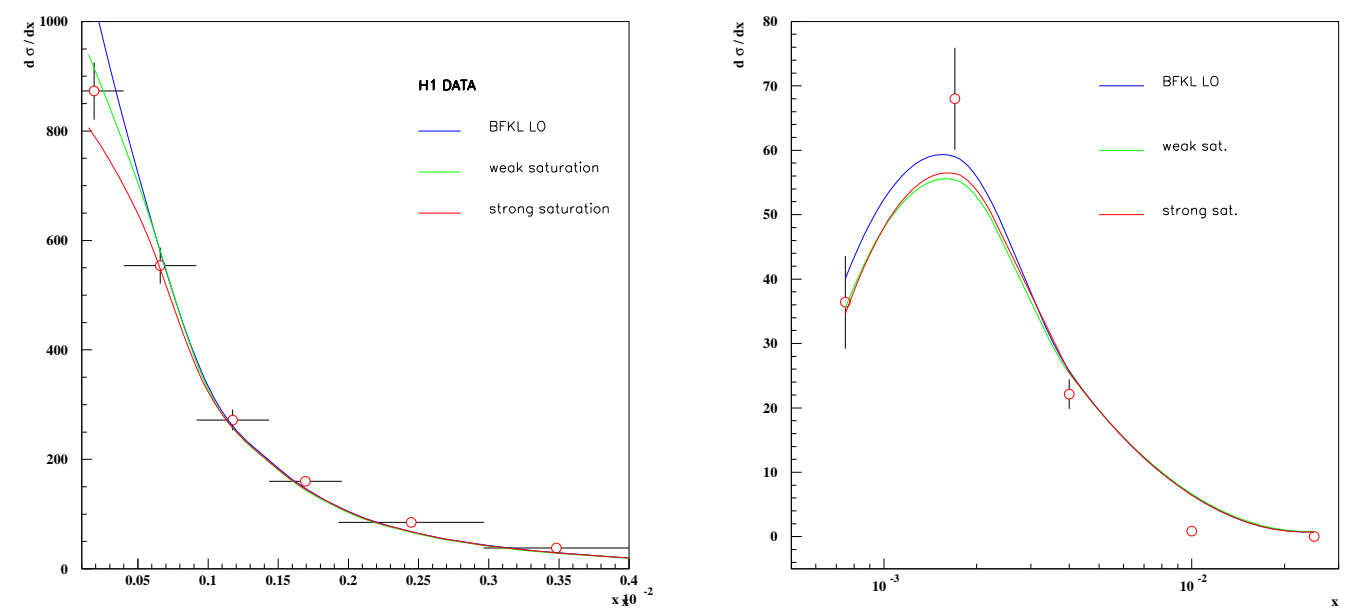

FIGURE 2. Comparisons between the H1 (left plot) and ZEUS (right plot) forward-jet new data for $d \sigma / d x$ and the BFKL and saturation parametrizations.

Let us now look at the new data [10, 11] for $d \sigma / d x$ which go to lower $x$. Without performing any new fit of the parameters, but rather by taking the values already obtained, the three parametrizations describe very well the new data, as shown on Fig2. One cannot really distinguish between the three curves even if at small values of $x$, one starts to see the difference between them. At the lowest values of $x$, NLOQCD predictions are about a factor 1.5 to 2.5 below the data depending on the experiment and the error bars. However, it could be that adding a resolved-photon component to the NLO predictions will pull them within the uncertainties which moderates the conclusion that the BFKL resummation is needed to describe those data. The fact that two saturation parametrizations are consistent with the data also asks for further study.

We intend to complete our analysis [12] by considering the other measurements $d \sigma / d Q^{2}$ and $d \sigma / d k_{T}$ by ZEUS and $d \sigma / d x d Q^{2} d k_{T}^{2}$, by H1 and Mueller-Navelet jets [13] at Tevatron or LHC. These could help clarifing the situation [14].

\section{REFERENCES}

1. L. N. Lipatov, Sov. J. Nucl. Phys. 23, (1976) 338; E. A. Kuraev, L. N. Lipatov and V. S. Fadin, Sov. Phys. JETP 45, (1977) 199; I. I. Balitsky and L. N. Lipatov, Sov. J. Nucl. Phys. 28, (1978) 822.

2. A. H. Mueller, Nucl. Phys. Proc. Suppl. B18C (1990) 125; J. Phys. G17 (1991) 1443.

3. L. V. Gribov, E. M. Levin and M. G. Ryskin, Phys. Rep. 100 (1983) 1.

4. K. Golec-Biernat and M. Wüsthoff, Phys. Rev. D59 (1999) 014017; Phys. Rev. D60 (1999) 114023.

5. C. Marquet Nucl. Phys. B705 (2005) 319.

6. H1 Collaboration, C. Adloff et al, Nucl. Phys. B538 (1999) 3.

7. ZEUS Collaboration, J. Breitweg et al, Eur. Phys. J. C6 (1999) 239.

8. J. G. Contreras, R. Peschanski and C. Royon, Phys. Rev. D62 (2000) 034006.

9. C. Marquet, R. Peschanski and C. Royon, Phys. Lett. B599 (2004) 236.

10. A. Knutsson for the H1 Collaboration, these proceedings.

11. ZEUS Collaboration, hep-ex/0502029, N. Vlasov for the ZEUS collaboration, these proceedings.

12. C. Marquet and C. Royon, in preparation.

13. A. H. Mueller and H. Navelet, Nucl. Phys. B282 (1987) 727.

14. C. Marquet and R. Peschanski, Phys. Lett. B587 (2004) 201; C. Marquet, hep-ph/0406111 